\title{
Wireless Power Transfer System for Mobile Robots via Magnetic Resonant Coupling with Impedance Matching
}

Wataru HIJIKATA ( $\nabla$ hijikata.w.aa@m.titech.ac.jp )

Tokyo Institute of Technology: Tokyo Kogyo Daigaku https://orcid.org/0000-0002-5433-1919

Toshiki Ohori

Tokyo Institute of Technology: Tokyo Kogyo Daigaku

Xiang Li

Laurel Bank Machines Co.Ltd.,

Hideyuki Nakanishi

Laurel Bank Machines Co Ltd.,

Shigeki Ozawa

Laurel Bank Machines Co. Ltd.,

\section{Research Article}

Keywords: Wireless power transfer, Mobile robot, Impedance matching, Coupling coefficient, Driver coil

Posted Date: January 10th, 2022

DOI: https://doi.org/10.21203/rs.3.rs-1229883/v1

License: (9) This work is licensed under a Creative Commons Attribution 4.0 International License.

Read Full License 


\title{
Wireless Power Transfer System for Mobile Robots via Magnetic Resonant Coupling with Impedance Matching
}

\author{
Toshiki Ohori ${ }^{1}$, Xiang $\mathrm{Li}^{2}$, Hideyuki Nakanishi², Shigeki Ozawa ${ }^{2}$, Wataru Hijikata ${ }^{1 *}$ \\ ${ }^{*}$ Correspondence: hijikata.w.aa@m.titech.ac.jp \\ ${ }^{1}$ Department of Mechanical Engineering, School of Engineering, Tokyo Institute of Technology, 2-12-1, Ookayama, \\ Meguro-ku, Tokyo 152-8550, Japan \\ ${ }^{2}$ LAUREL BANK MACHINES CO., LTD., 1-1-2, Toranomon, Minato-ku, Tokyo 105-8414, Japan
}

\begin{abstract}
Wireless power transfer via magnetic resonant coupling can be used to supply power to a mobile robot within a few meters of a transmitter coil. However, when the robot moves or its power consumption fluctuates, its input impedance varies and causes power reflection. Therefore, we propose the use of a driver coil on the transmitter side to match the input impedance. The input impedance is matched and power reflection is eliminated by regulating the coupling coefficient between the driver and the transmitter. During experiments, the transmitting efficiency showed good agreement with the calculated value, and the input impedance was matched under varying distances and load resistances. Therefore, the proposed system was demonstrated to solve the power reflection problem in mobile robots.
\end{abstract}

Key Words: Wireless power transfer, Mobile robot, Impedance matching, Coupling coefficient, Driver coil

\section{Introduction}

In recent years, various unmanned mobile robots, such as automated guided vehicles and autonomous mobile robots (AMRs), have been widely studied and developed [1-5]. In particular, AMRs that fly near the ceiling, including drones, could be used for automatic surveillance in warehouses to eliminate the blind spots of surveillance cameras [6] or for serving customers indoors to make effective use of space [7, 8]. However, in both these applications, the power supply method remains an unsolved problem because the robot must fly continuously over a wide area. A wired power supply is limited by the cable length and the risk of tangling the cable. Further, a battery-only power supply is limited by the battery capacity, and the larger the capacity gets, the heavier the battery gets. Therefore, these power supply methods are undesirable for AMRs. As an alternative, wireless power transfer (WPT) is considered an effective power supply method, and is being actively researched $[9,10]$.

WPT is of two main types: radiative and nonradiative [11]. This study uses magnetic resonance coupling, a type of nonradiative transfer. In this method, inductor and capacitor (LC) circuit resonance is used to transmit power over longer distances than in the case of inductive coupling alone [12]. This approach is being investigated for applications such as power supply for electric vehicles (EVs) and implantable medical devices like artificial hearts [13-17].

As shown in Fig.1, this study proposes a system in which several transmitter coils are installed in the ceiling at constant intervals to supply power, and the receiver coil is mounted on the flying robot. The robot then receives power from the nearest transmitter coil.

Most existing and proposed technologies for applications such as power supply for EVs during the stoppage period [18-20] assume conditions in which the distance between the transmitter and receiver coils is almost constant. The distance between coils changes with vertical and horizontal movements in WPT for mobile robots. Further, fluctuations in power consumption are predicted; these will change the input impedance of the circuit and make it unequal to the output impedance, resulting in power reflection as shown in Fig.2. Increased power reflection reduces the power delivered to the later part of the circuit [21]. In a previous study, impedance matching was performed by controlling the output voltage (i.e., duty cycle) of the DC-DC converter [22]. Although this method reduces the reflected power when the load resistance is lower than the optimal point, it is not applicable when the load requires a constant voltage drive and the matching range is narrow. An impedance matching circuit with switching has also been proposed [23]. Although it realizes almost completely automatic impedance matching with changes in the distance between the coils, it requires numerous capacitors and multiple coils to change the capacitance and inductance, and the matching accuracy is not high in some cases.

To solve these problems, this study proposes a novel system that can perform more effective impedance matching with both changes in the distance between coils and in the load resistance. Installing an additional mechanism that could increase the weight of the flying robot is undesirable. Therefore, we add another coil to the transmitter side and control its position to match the impedance. Based on this principle, we have designed and fabricated coils to achieve high output power over a wide area and experimentally evaluated the effect of impedance matching in our proposed system.

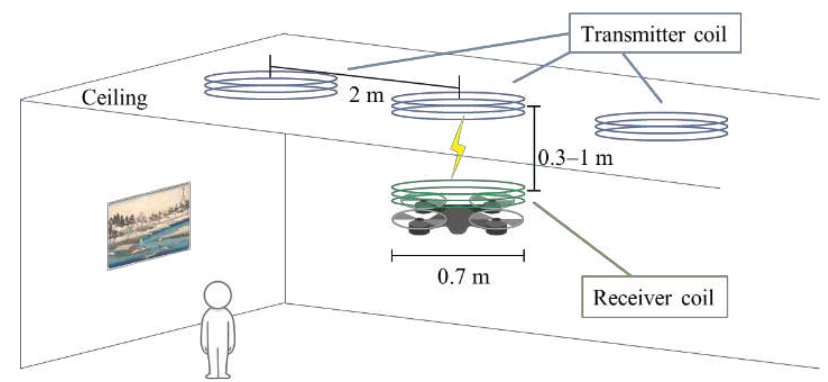

Fig.1 Expected usage environment

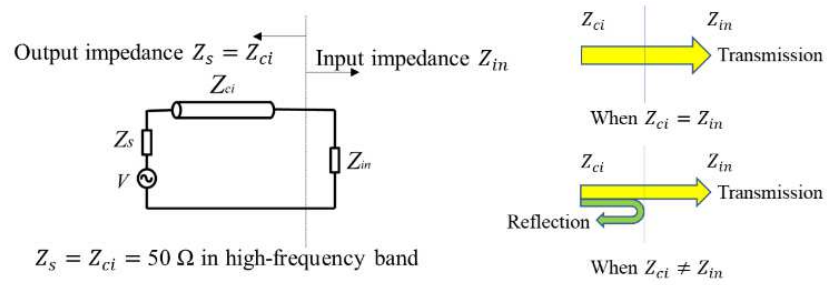

Fig. 2 Condition under which power reflection occurs 


\section{Principle of proposed impedance matching}

Fig. 3 shows the circuit diagram of a conventional WPT system. In this figure, $V$ is the input voltage to the circuit; $Z_{s}$, the output impedance of the power source; $R_{\text {回}}$, the load resistance; $k_{12}$, the coupling coefficient between the coils; $L_{\square}$, the self-inductance of the coil; $C_{\text {目}}$, the capacitance of the capacitor; $R_{\text {回}}$, the parasitic resistance of each circuit; and $I_{\text {巴] }}$, the current flowing through each circuit. In all these notations, $i=1$ or 2 , respectively, indicating the transmitter side or receiver side. This two-coil system affords the advantage of thinner and lighter coils; however, the impedance fluctuates greatly, resulting in power reflection. In the high-frequency band, the output impedance of the equipment and the characteristic impedance of the transmission line are designed to be $50 \Omega$. To maximally use the capability of the power source, the input impedance of the whole circuit should be matched to $50 \Omega$.

In this study, we propose an impedance matching method with a driver coil that can be applied for addressing both coil misalignment and load fluctuation. As shown in Fig.4, the driver coil is installed over the transmitter coil, and its position is controlled by an actuator in the horizontal direction to change the coupling coefficient $k_{01}$ between itself and the transmitter coil to match the impedance. Fig.5 shows the circuit diagram of the proposed system, and Fig. 6 shows its T-type equivalent circuit. Note that the subscript 0 indicates the driver coil circuit. For simplicity, the cross-coupling between the driver coil and the receiver coil is ignored. Under the condition that the resonant frequencies $\omega$ of all circuits are the same, the impedances $Z_{0}, Z_{1}$ and $Z_{2}$ can be expressed as follows:

$$
\begin{gathered}
\omega=\frac{1}{\sqrt{L_{0} C_{0}}}=\frac{1}{\sqrt{L_{1} C_{1}}}=\frac{1}{\sqrt{L_{2} C_{2}}} \\
Z_{2}=R_{2}+R_{L} \\
Z_{1}=R_{1}+\frac{\left(\omega k_{12} \sqrt{L_{1} L_{2}}\right)^{2}}{R_{2}+R_{L}} \\
Z_{0}=R_{0}+\frac{\left(\omega k_{01} \sqrt{L_{0} L_{1}}\right)^{2}}{Z_{1}}
\end{gathered}
$$

$Z_{1}$ is the input impedance of the two-coil system, and it varies with the position and power consumption of the robot. In other words, because $k_{12}$ varies with the distance between the transmitter and the receiver coils and $R_{\text {曰 }}$ varies with the state of the load, $Z_{1}$ inevitably varies in mobile robots. However, the input impedance $Z_{0}$ in the proposed three-coil system can be kept constant by varying the coupling coefficient $k_{01}$. When $Z_{1}$ becomes larger, the driver coil is moved to overlap the transmitter coil more to increase $k_{01}$, and vice versa. For example, when the coupling coefficient changes depending on the distance between the transmitter and the receiver coils, the driver coil is moved as shown in Fig.7(a), and when the load resistance changes, the driver coil is moved as shown in Fig.7(b). Notably, the driver coil must be designed to achieve the required range of $k_{01}$ to match the impedance of the system.

Considering the impedance of each part and the fact that only the parasitic resistance reduces the transmitting efficiency, the three-coil system is expressed as follows:

$$
\eta=\eta_{0} \eta_{1} \eta_{2}=\frac{Z_{0}-R_{0}}{Z_{0}} \times \frac{Z_{1}-R_{1}}{Z_{1}} \times \frac{Z_{2}-R_{2}}{Z_{2}}
$$

Compared to the two-coil system, the three-coil system is smaller by $\eta_{0}$; however, the effect can be almost ignored if the parasitic resistance $R_{0}$ is small enough for $Z_{0}=50 \Omega$. In this study, the transmitting efficiency is defined as the ratio of the received power at the load resistance to the active power input to the circuit.

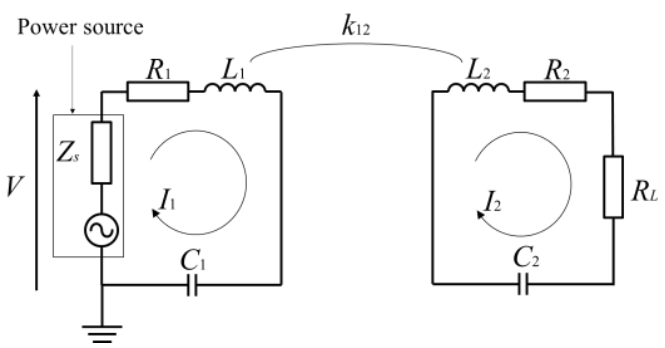

Fig.3 Circuit of two-coil system with magnetic resonant coupling

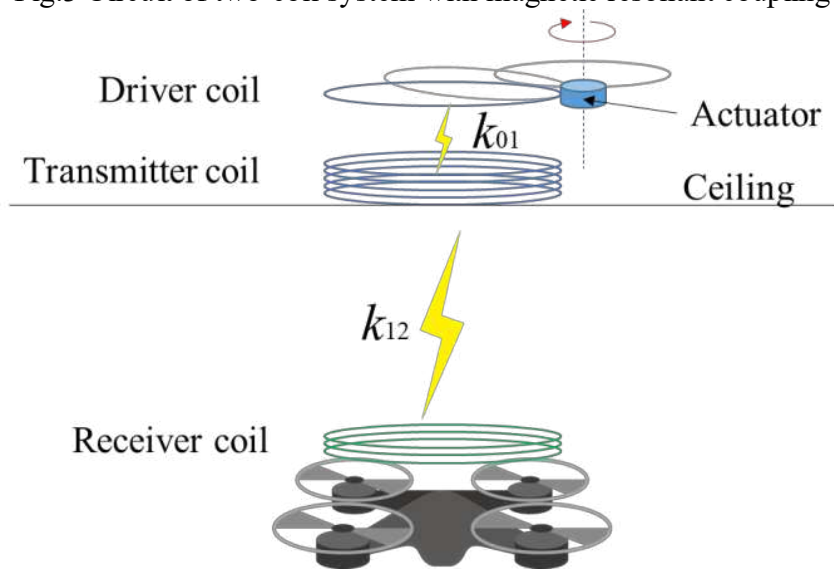

Fig.4 Arrangement of proposed coils

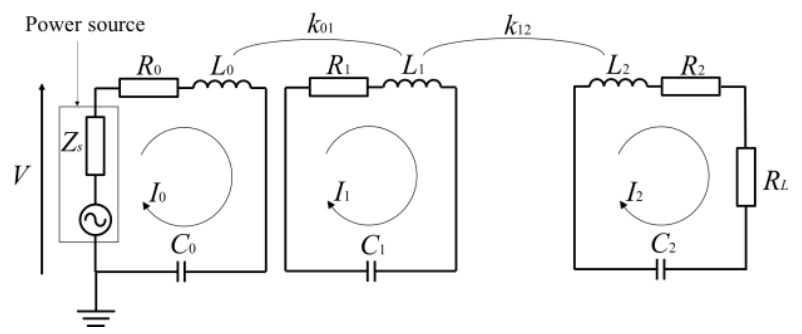

Fig.5 Equivalent circuit of three-coil system with driver coil

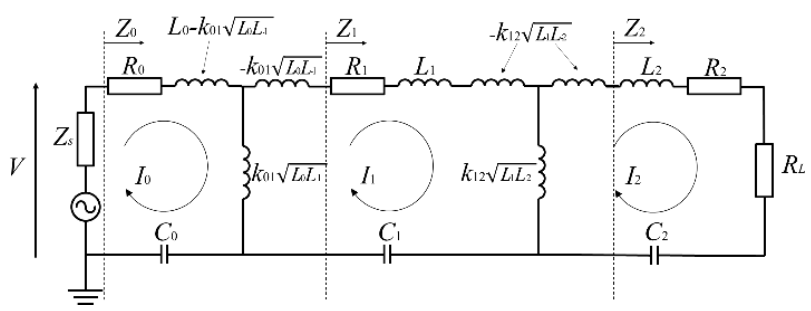

Fig.6 T-type equivalent circuit of three-coil system 

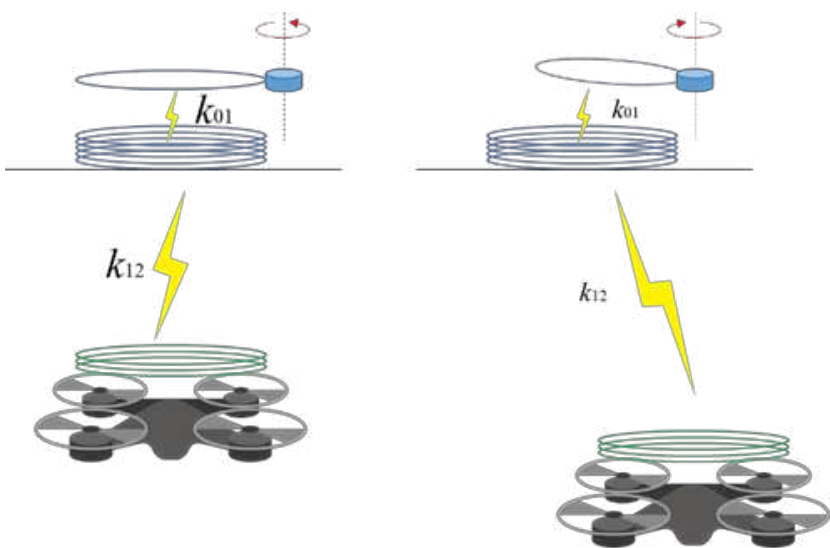

(a) When distance changes
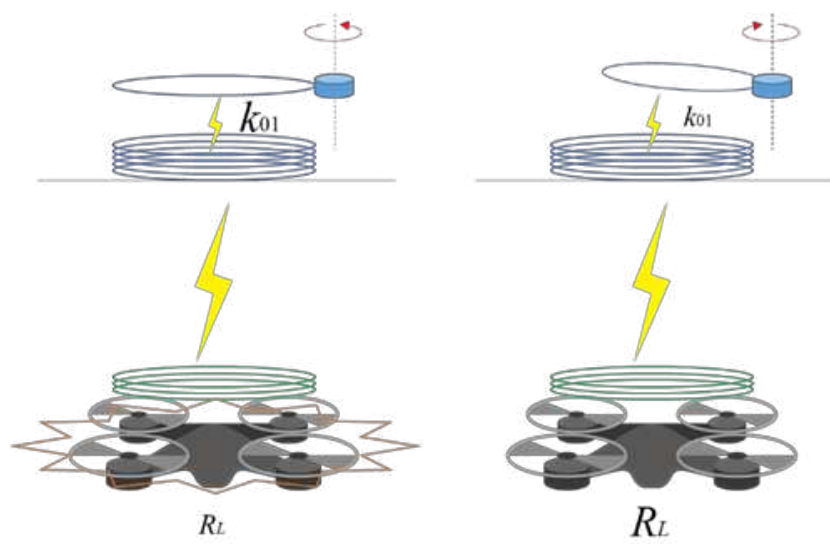

(b) When load resistance changes

Fig.7 Movement of driver coil

\section{System design System overview}

Fig. 8 shows an overview of the entire system. On the transmitter side, the signal from the function generator was amplified by the power amplifier and fed into the circuit using magnetic resonant coupling. The energy transferred to the receiver side was converted to a predetermined DC voltage through a rectifying/smoothing circuit and a DC-DC converter. Moreover, it was used to float the robot, charge the battery, etc. In addition, the reflected power was measured from the information of the driver coil circuit, and the optimal position of the driver coil was estimated and controlled so that the reflection becomes smaller. However, to verify the principle, the driver coil was set to the optimal position by moving it manually.

Further, we designed the coils assuming a power supply frequency of $150 \mathrm{kHz}$. All coils were assumed to be parallel, and the power losses in the robot material and after the rectifier were not considered. The rated output of the power amplifier was $100 \mathrm{~W}$ at this time, and we aimed to supply $20 \mathrm{~W}$ over a wide area and a maximum instantaneous power of $50 \mathrm{~W}$.

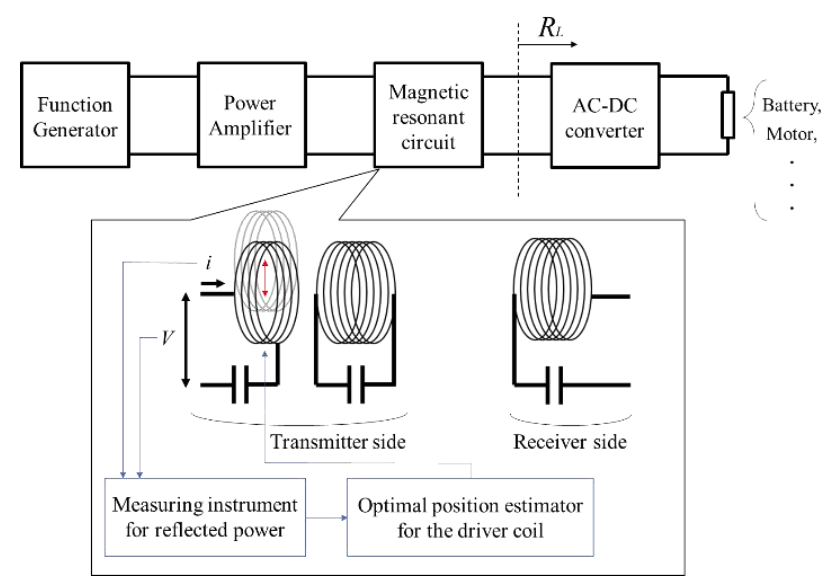

Fig.8 Proposed configuration for WPT

\section{Coil design}

For coil design, we ignored the power losses in the AC-DC conversion and the metal surface of the robot; therefore, all efficiencies were derived using Equation (5).

The dimensions and shapes of the transmitter and receiver coils were determined as follows. The coil diameters were set to the largest possible value, $D_{\text {曰 }}=D_{r}=0.7 \mathrm{~m}$, based on the space behind the ceiling and the robot size. In addition, we decided to use solenoid coils to keep the coil diameter large, because a coil with a larger diameter can generally transmit power over a longer distance. The receiver coil had strict weight and thickness limits because it was mounted on the flying robot. Therefore, we first determined the parameters of the receiver coil and then determined those of the transmitter coil to achieve the appropriate transmission efficiency. The coil pitch was determined to be two times the wire diameter; this was optimal considering the quality factor of the coil [24]. We also used the calculation model for the inductance and coupling coefficient of the coil by the filament method [25] and the calculation model for the parasitic resistance of the coil [26]. We used multilayer ceramic capacitors (MLCCs) as they have low parasitic resistance. Preliminary experiments suggested that their parasitic resistances were $0.5 \Omega$ on average; therefore, we used this value in the simulation

For the receiver coil, the weight and thickness limits were respectively $60 \mathrm{~g}$ and $50 \mathrm{~mm}$. We then set the number of turns $N_{\text {曰 }}=$ 15 and the wire diameter $d_{\text {巴 }}=0.4 \mathrm{~mm}$ to achieve the maximum quality factor in a range in which the wire diameter was not too small to avoid making it difficult to wind the coil. Under these conditions, the distance between the centers of the coils was defined as $[x, 0, z]$, as shown in Fig.9, and the transmitter coil was designed to have a transmitting efficiency of more than $40 \%$ at a distance of $[0 \mathrm{~m}, 0 \mathrm{~m}$, $1.0 \mathrm{~m}$ ] when the load resistance was $10 \Omega$. Equation (5) with $\eta_{0}=1$ was used for calculating the efficiency. The transmitter coil had no weight or thickness limits; nonetheless, extreme values are not necessary. Therefore, we selected the combination in which the number of turns was $N_{\square}=30$ and wire diameter was $d_{\square}=1.5 \mathrm{~mm}$ from Fig. 10.

For the driver coil, we set the coil diameter $D_{\text {曰 }}=0.7 \mathrm{~m}$ and the wire diameter $d_{\text {曰 }}=1.5 \mathrm{~mm}$ to maintain consistency with the transmitter coil. In this case, the coupling coefficient $k_{01}$ between the driver coil and the transmitter coil was the maximum when they were completely overlapped, and it became smaller when the driver 
coil was moved sideways. For a 5-mm gap between the driver and transmitter coils, the magnitude of the coupling coefficient $k_{01}$ was calculated to be approximately in the range of $0-0.5$ regardless of the number of turns of the driver coil. Solving Equation (4) for the magnitude of $k_{01}$ gives

$$
\left|k_{01}\right|=\frac{1}{\omega} \sqrt{\frac{\left(Z_{0}-R_{0}\right) Z_{1}}{L_{0} L_{1}}}
$$

The $L_{0}$ and $R_{0}$ values were calculated based on the number of turns of the driver coil; therefore, the number of turns should be decided to satisfy $\left|k_{01}\right| \leq 0.5$ at $0.3 \leq z \leq 1 \mathrm{~m}$, which is the flight range of the robot, when $Z_{0}$ is matched at $50 \Omega$. It is sufficient to assume the maximum value of $Z_{1}$; therefore, we assumed that $R_{\square}$ is the minimum from Equation (3). $R_{\text {曰 }}$ is the resistance value when looking at the later part of the circuit from the front of the AC-DC converter, as shown in Fig.8. Assuming that $R_{\text {[回回 }}$ is the load resistance of the battery, motor, etc., $R_{\text {回 }}$ is calculated as

$$
R_{\text {曰 }}=\frac{R_{\text {out }}}{D^{2}} \times \frac{1}{1.347}={V_{\text {in }}}^{2} \times \frac{R_{\text {out }}}{V_{\text {out }}{ }^{2}} \times \frac{1}{1.347}
$$

where $D$ is the conduction ratio of the DC-DC converter, and the coefficient is the theoretical impedance transformation ratio when a diode bridge rectifier circuit is used [27]. The DC-DC converter used in the system (UWE-12/10-Q48N-C, Murata) has an output voltage of $V_{\text {out }}=12 \mathrm{~V}$ and input voltage of $V_{\text {in }}=18-75 \mathrm{~V}$; the robot's maximum power usage $P_{r}$ is $50 \mathrm{~W}$. Therefore, the minimum value of $R_{\text {曰 }}$ was obtained as $4.8 \Omega$. Under this situation, when the number of turns of the driver coil $N_{\square}=10$, the coupling coefficient $k_{01}$ required for impedance matching satisfies the condition as shown in Fig.11.
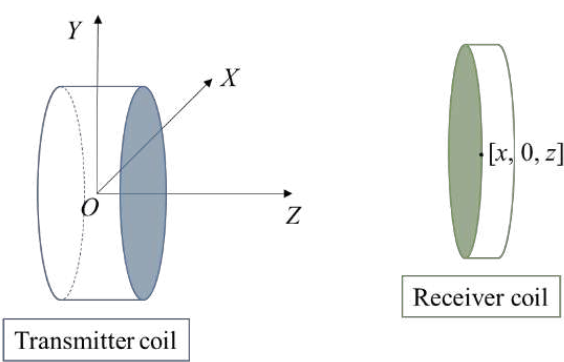

Fig.9 Definition of distance between coils

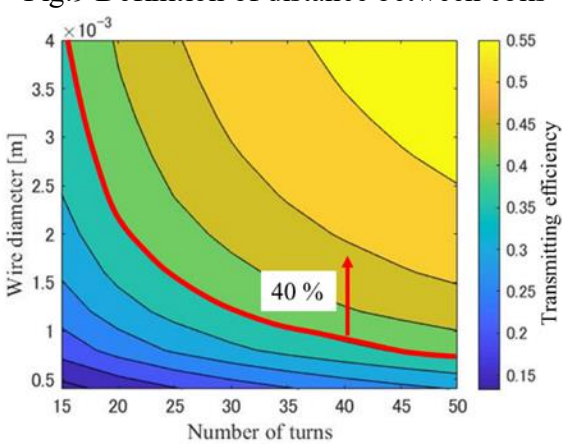

Fig.10 Transmitting efficiency against parameters of transmitter coil

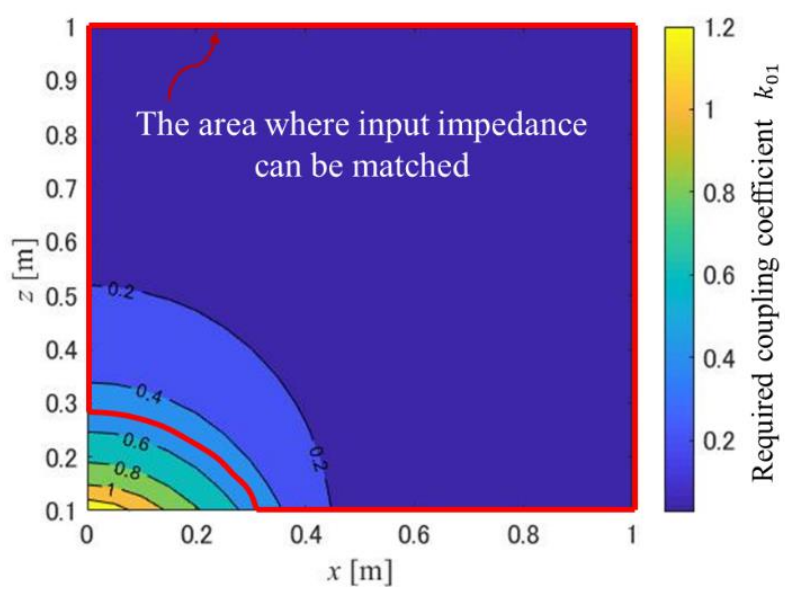

Fig.11 Simulation results of required coupling coefficient $k_{01}$ for $R_{\text {回 }}=4.8 \Omega$

\section{Simulation of transmitting efficiency}

Fig.12 shows simulation results of the transmitting efficiency versus the position of the receiver coil when the load resistance is 10 $\Omega$. The efficiency exhibited a valley in the graph because the direction of the magnetic flux to the receiver coil changed depending on the position of the coils, as shown in Fig.13. When the direction was switched, the sum of the magnetic flux through the receiver coil as well as the efficiency were zero. Nonetheless, the simulation revealed high efficiency over a sufficiently wide area.

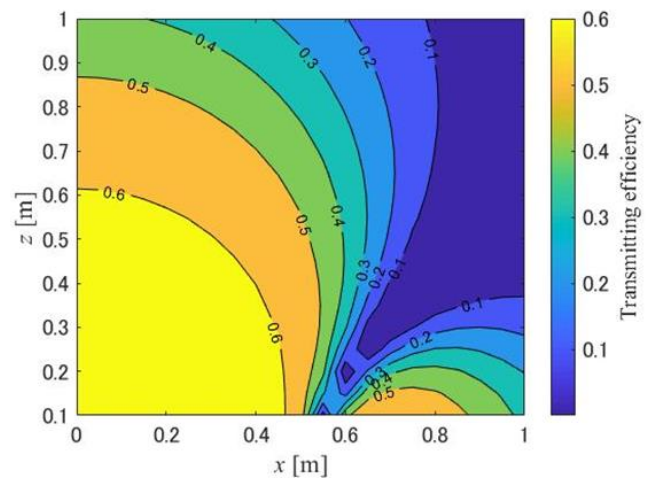

Fig.12 Simulation results of transmitting efficiency for $R_{\text {回 }}=10 \Omega$

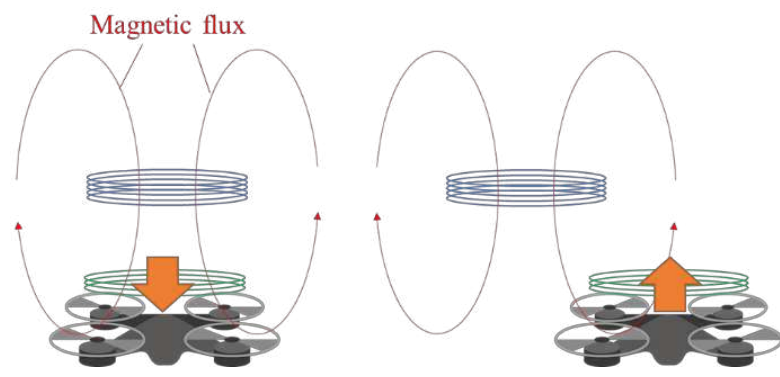

Fig.13 Direction of magnetic flux

\section{Prototyping}

Fig.14(a) shows the prototype coils. When the center of the receiver coil was hollowed out, it weighed only $50 \mathrm{~g}$ and had a thickness of approximately $2 \mathrm{~mm}$. A vinyl-insulated stranded wire was used for the driver and the transmitter coil, and a solid wire was used for the receiver coil. In addition, leaded MLCCs (RDE series, 
Murata) were used for the capacitors. Considering the withstand voltage, the capacitor for the transmitter circuit was made as shown in Fig. 14(b), and the others were produced similarly.
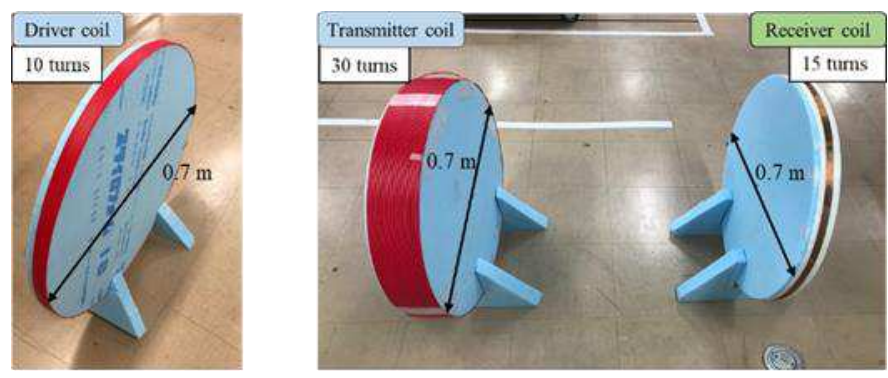

(a) Coils

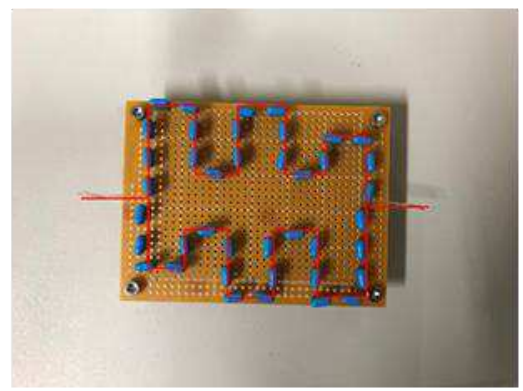

(b) Capacitor for transmitter circuit

Fig.14 Prototype developed in this study

\section{Experiments, results, and discussions}

In the following experiments, a function generator (AFG-2125, GW Instek), a voltage follower (when power was low), and a power amplifier (T145-5027A, Thamway; when power was high) were used in the power supply system. In addition, a DC-DC converter was not used for simplicity. Because iron and other metals near the coils would adversely affect the power transmission, the coils were placed to avoid them.

\section{Measurements of efficiency and impedance}

By using the prototype coils, we measured the efficiency and impedance to evaluate the validity of the proposed system. First, the coil positions were adjusted manually. Further, the experiment was performed at low power $(<1 \mathrm{~mW})$ for safety reasons. When the resonant frequency was shifted, the power supply frequency was adjusted to achieve a perfect resonant state and power factor of 1 . In this experiment, the transmitting efficiency and input impedance were measured for the lateral displacement of the receiver coil (load resistance: $10 \Omega$ ) and the load variation $(x=0 \mathrm{~m})$ at $z=0.5 \mathrm{~m}$. The input impedance was also measured in the two-coil system without the impedance matching mechanism. Fig. 15 shows a demonstration of the matching operation when a light bulb was connected as a load.

Figs. 16 and 17 respectively show the measurement results for the lateral displacement and load variation. Good agreement was seen between the simulated and measured transmitting efficiency. As in Fig.12, the efficiency exhibited a valley in Fig.16(a). The results in Figs.16(b) and 17(b) confirmed the effectiveness of the proposed impedance matching system and indicated that the input impedance of the three-coil system can be matched to approximately $50 \Omega$.

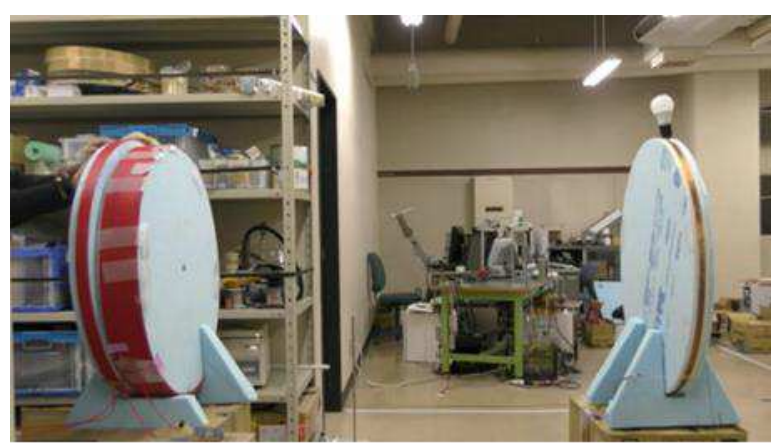

(a) Without matching

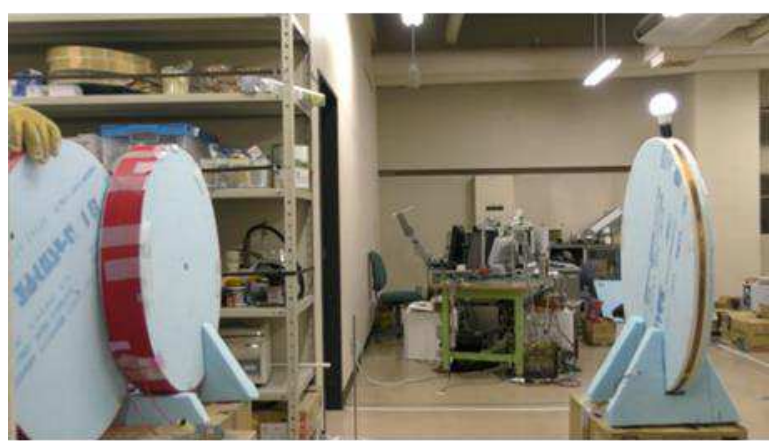

(b) With matching by driver coil

Fig.15 Demonstration of impedance matching system

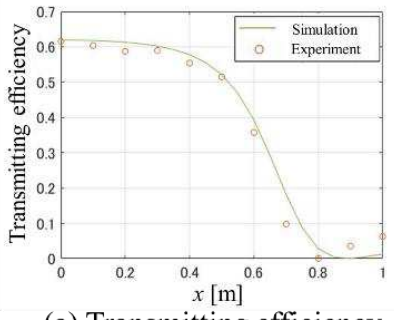

(a) Transmitting efficiency

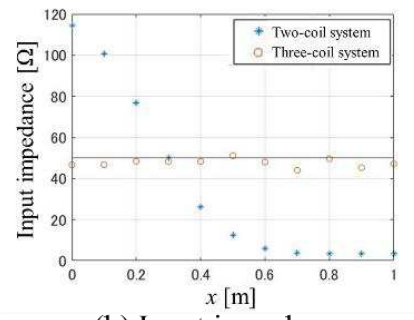

(b) Input impedance
Fig.16 Measured results for $R_{\square}=10 \Omega$ at $[x, 0,0.5 \mathrm{~m}]$

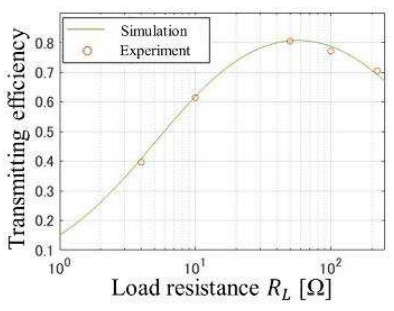

(a) Transmitting efficiency

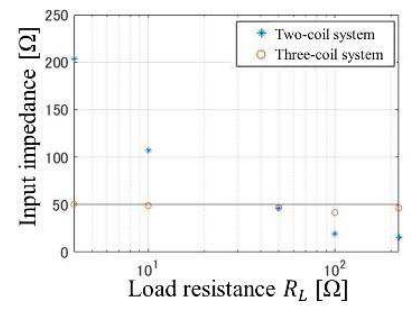

(b) Input impedance
Fig.17 Measured results for variable $R_{\text {曰 }}$ at $[0,0,0.5 \mathrm{~m}]$

\section{Comparison of received power}

We experimentally compared the received power between the twoand three-coil systems when the output power of the power source was kept constant at $10 \mathrm{~W}$. The received power of the three-coil system was estimated to be higher because the input power to the circuit was reduced by the reflected power in the two-coil system. A noninductive resistor of $10 \Omega$ was used as the load. Under these conditions, we measured the received power at 24 points with $0.2-\mathrm{m}$ intervals in the range of $x=0-1.0 \mathrm{~m}$ and $z=0.4-1.0 \mathrm{~m}$.

Fig. 18 shows the measurement results. All data were normalized 
by the received power of the two-coil system at $[x, 0, z]=[0 \mathrm{~m}, 0 \mathrm{~m}$, $1.0 \mathrm{~m}]$. The data with the same amount of lateral displacement were plotted by lines. This graph shows that the received power of the three-coil system was higher than that of the two-coil system over a wide range. At the point where the received power of both systems was almost the same, such as $[x, 0, z]=[0 \mathrm{~m}, 0 \mathrm{~m}, 0.6 \mathrm{~m}]$, the input impedance can be assumed to be $50 \Omega$ even for the two-coil system.

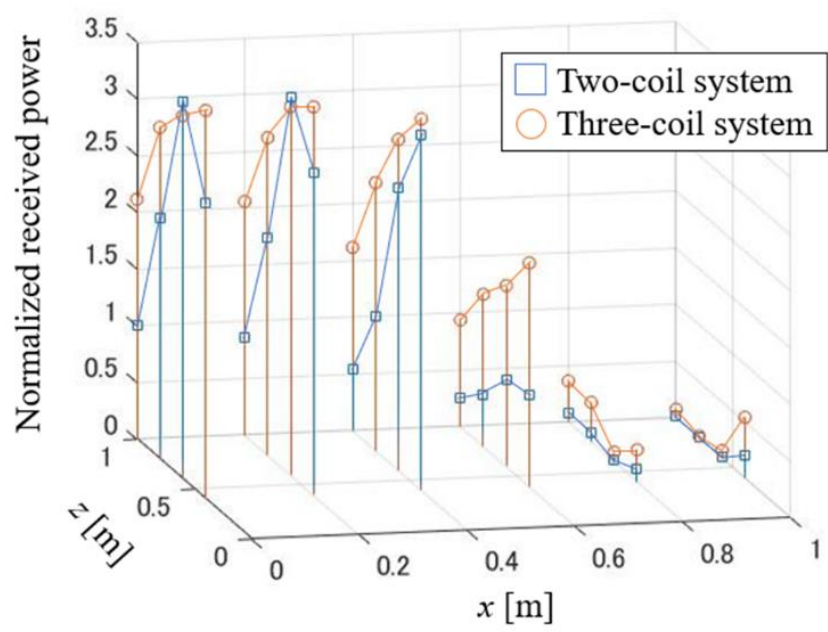

Fig.18 Comparison of normalized received power between two-coil and three-coil system

\section{High-power transmission}

The above-described experiments revealed that the impedance matching mechanism of the proposed system was effective over a wide range. The effect was particularly noticeable for a large distance between the coils. Accordingly, we experimentally tested whether 20 $\mathrm{W}$ can be transmitted when the coil was displaced laterally at $z=1$ $\mathrm{m}$. For safety reasons, we took the data when the received power exceeded $20 \mathrm{~W}$ or when the output power of the power source reached $100 \mathrm{~W}$. We used a load resistance of $10 \Omega$ and performed measurements at $0.1 \mathrm{~m}$ intervals in the range of $x=0-0.5 \mathrm{~m}$ as this was considered to have a transmitting efficiency above $20 \%$ from Fig.12.

Fig. 19 shows the experimental results. The system could supply 20 $\mathrm{W}$ at distances of up to $x=0.3 \mathrm{~m}$, thus confirming that it can supply power over a sufficiently wide area. At the same time, the system could not supply power at distances of $x=0.4$ and $0.5 \mathrm{~m}$. This might be partly because most of the power was consumed as heat in the parasitic resistance of the coils and capacitors. This heat can increase the resistance of the conductor, resulting in additional heat loss, and change the capacitance of the capacitor, resulting in a deviation from the resonant state. If heat is the cause of the efficiency loss, an environment that facilitates heat dissipation must be created.

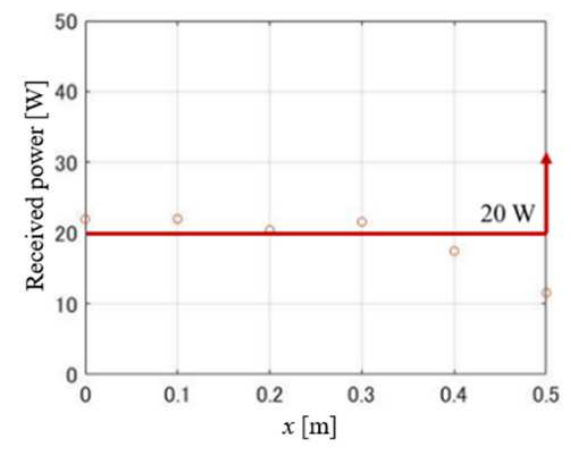

Fig.19 Received power for $R_{\text {马 }}=10 \Omega$ at $[x, 0,1.0 \mathrm{~m}]$

The efficiency can be improved in other ways. In the frequency range of $100-200 \mathrm{kHz}$, the use of a Litz wire has been reported to reduce the skin effect and decreases the parasitic resistance of the coil [28], thereby improving the efficiency. In addition, the incorporation of ferrite would not only increase the coupling coefficient between the coils but also provide a shielding effect to reduce external influences [29, 30].

\section{Conclusions}

We proposed a system that can match the impedance by the driver coil with changes in both the distance between coils and the load resistance to solve the impedance mismatch problem in WPT due to the state of mobile robots. Simulation and experimental results confirmed that impedance matching could be achieved by moving the driver coil for mitigating the load fluctuation and relative displacement between the transmitter and the receiver coils in the axial and horizontal directions.

In this study, the driver coil was set to the optimal position by moving it manually to verify the principle. In a future study, we will design a system that can automatically control the position of the driver coil based on the measured reflected power.

\section{Abbreviations}

AMR: Autonomous mobile robot; WPT: Wireless power transfer; MLCC: Multilayer ceramic capacitor.

\section{Acknowledgements}

Thanks to everyone who was involved in this research or provided valuable comments on it. We would also like to thank Editage (www.editage.com) for English language editing.

\section{Author's contributions}

TO proposed the method described in this paper, conducted all simulations and experiments, and drafted the manuscript. XL, HN and SO provided advice, conducted some of the experiments, and checked and revised the manuscript. WH proposed the method described in this paper, provided advice, conducted some of the experiments, and checked and revised the manuscript. All authors read and approved the final manuscript.

\section{Funding}

This study was funded by LAUREL BANK MACHINES CO. LTD. 


\section{Availability of data and materials}

Not applicable.

\section{Declarations}

This study was funded by LAUREL BANK MACHINES CO. LTD.

\section{Competing interests}

The authors declare that they have no competing interests.

\section{References}

1. Oyekanlu EA, Smith AC, Thomas WP, et al (2020) A Review of Recent Advances in Automated Guided Vehicle Technologies: Integration Challenges and Research Areas for 5G-Based Smart Manufacturing Applications. IEEE Access 8:202312-202353. https://doi.org/10.1109/ACCESS.2020.3035729

2. Ardiny H, Witwicki S, Mondada F (2015) Construction Automation with Autonomous Mobile Robots: A Review. Int Conf Robot Mechatronics, ICROM 2015 418-424. https://doi.org/10.1109/ICRoM.2015.7367821

3. Alatise MB, Hancke GP (2020) A Review on Challenges of Autonomous Mobile Robot and Sensor Fusion Methods. IEEE Access 8:39830-39846. https://doi.org/10.1109/ACCESS.2020.2975643

4. Adzhar N, Yusof Y, Ahmad MA (2020) A Review on Autonomous Mobile Robot Path Planning Algorithms. Adv Sci Technol Eng Syst 5:236-240. https://doi.org/10.25046/aj050330

5. Mohanty P, Parhi D (2013) Controlling the Motion of an Autonomous Mobile Robot Using Various Techniques: a Review. J Adv Mech Eng 24-39. https://doi.org/10.7726/jame.2013.1003

6. Hell PM, Varga PJ (2019) Drone Systems for Factory Security and Surveillance. Interdiscip Descr Complex Syst 17:458-467. https://doi.org/10.7906/indecs.17.3.4

7. Mao W, Zhang Z, Qiu L, et al (2017) Indoor Follow Me Drone. MobiSys 2017 - Proc 15th Annu Int Conf Mob Syst Appl Serv 345-358. https://doi.org/10.1145/3081333.3081362

8. Fuhrman T, Schneider D, Altenberg F, et al (2019) An Interactive Indoor Drone Assistant. IEEE Int Conf Intell Robot Syst 6052-6057. https://doi.org/10.1109/IROS40897.2019.8967587

9. Zhang Z, Pang H, Georgiadis A, Cecati C (2019) Wireless Power Transfer - An Overview. IEEE Trans Ind Electron 66:1044-1058. https://doi.org/10.1109/TIE.2018.2835378

10. Okoyeigbo O, Olajube AA, Shobayo O, et al (2021) Wireless power transfer: A review. IOP Conf Ser Earth Environ Sci 655:. https://doi.org/10.1088/17551315/655/1/012032

11. Shadid R, Noghanian S, Nejadpak A (2016) A Literature Survey of Wireless Power Transfer. IEEE Int Conf Electro Inf Technol 2016-Augus:782-787. https://doi.org/10.1109/EIT.2016.7535339

12. Kurs A, Karalis A, Moffatt R, et al (2007) Wireless Power Transfer via Strongly Coupled Magnetic Resonances.
Science (80- ) 317:83-86.

https://doi.org/10.1126/science.1143254

13. Bi Z, Kan T, Mi CC, et al (2016) A review of wireless power transfer for electric vehicles: Prospects to enhance sustainable mobility. Appl Energy 179:413-425. https://doi.org/10.1016/j.apenergy.2016.07.003

14. Sun L, Ma D, Tang H (2018) A review of recent trends in wireless power transfer technology and its applications in electric vehicle wireless charging. Renew Sustain Energy Rev 91:490-503. https://doi.org/10.1016/j.rser.2018.04.016

15. Kim JD, Sun C, Suh IS (2014) A Proposal on Wireless Power Transfer for Medical Implantable Applications Based on Reviews. IEEE Wirel Power Transf Conf 2014, IEEE WPTC 2014 166-169. https://doi.org/10.1109/WPT.2014.6839592

16. Khan SR, Pavuluri SK, Cummins G, Desmulliez MPY (2020) Wireless Power Transfer Techniques for Implantable Medical Devices: A Review. Sensors (Switzerland) 20:1-58. https://doi.org/10.3390/s20123487

17. Hijikata W, Suzuki H (2019) Real-time Optimization of Coil Positioning in Wireless Power Transfer System for Artificial Heart. 2018 IEEE Wirel Power Transf Conf WPTC 2018 1-4. https://doi.org/10.1109/WPT.2018.8639256

18. Nguyen TD, Li S, Li W, Mi CC (2014) Feasibility Study on Bipolar Pads for Efficient Wireless Power Chargers. Conf Proc - IEEE Appl Power Electron Conf Expo - APEC 1676-1682. https://doi.org/10.1109/APEC.2014.6803531

19. Gao Y, Duan C, Oliveira AA, et al (2017) 3-D Coil Positioning Based on Magnetic Sensing for Wireless EV Charging. IEEE Trans Transp Electrif 3:578-588. https://doi.org/10.1109/TTE.2017.2696787

20. Khan N, Matsumoto H, Trescases O (2020) Wireless Electric Vehicle Charger With Electromagnetic Coil-Based Position Correction Using Impedance and Resonant Frequency Detection. IEEE Trans Power Electron 35:7873-7883. https://doi.org/10.1109/TPEL.2020.2965476

21. Kusaka K, Itoh JI (2012) Proposal of Switched-mode Matching Circuit in Power Supply for Wireless Power Transfer Using Magnetic Resonance Coupling. Conf Proc IEEE Appl Power Electron Conf Expo - APEC 653-660. https://doi.org/10.1109/APEC.2012.6165888

22. Moriwaki Y, Imura T, Hori Y (2011) Basic Study on Reduction of Reflected Power Using DC/DC Converters in Wireless Power Transfer System via Magnetic Resonant Coupling. INTELEC, Int Telecommun Energy Conf 0-4. https://doi.org/10.1109/INTLEC.2011.6099737

23. Beh TC, Kato M, Imura T, et al (2013) Automated Impedance Matching System for Robust Wireless Power Transfer via Magnetic Resonance Coupling. IEEE Trans Ind Electron 60:3689-3698. https://doi.org/10.1109/TIE.2012.2206337

24. Sampath JPK, Alphones A, Shimasaki H (2017) Coil design Guidelines for High Efficiency of Wireless Power Transfer (WPT). IEEE Reg 10 Annu Int Conf Proceedings/TENCON 1:726-729. https://doi.org/10.1109/TENCON.2016.7848098 
25. Babic SI, Akyel C (2008) Calculating Mutual Inductance Between Circular Coils With Inclined Axes in Air. IEEE Trans Magn 44:1743-1750.

https://doi.org/10.1109/TMAG.2008.920251

26. Kim J, Park YJ (2015) Approximate Closed-Form Formula for Calculating Ohmic Resistance in Coils of Parallel Round Wires with Unequal Pitches. IEEE Trans Ind Electron 62:3482-3489.

https://doi.org/10.1109/TIE.2014.2370943

27. Ohira $\mathrm{T}$ (2013) Power efficiency and optimum load formulas on RF rectifiers featuring flow-angle equations. IEICE Electron Express 10:1-9.

https://doi.org/10.1587/elex.10.20130230

28. Cho J, Sun J, Kim H, et al (2017) Coil Design for $100 \mathrm{KHz}$ and 6.78 MHz WPT system :Litz and Solid Wires and Winding Methods. IEEE Int Symp Electromagn Compat 803-806. https://doi.org/10.1109/ISEMC.2017.8077977

29. Kim J, Kim J, Kong S, et al (2013) Coil Design and Shielding Methods for a Magnetic Resonant Wireless Power Transfer System. Proc IEEE 101:1332-1342. https://doi.org/10.1109/JPROC.2013.2247551

30. Elnail KEI, Huang X, Xiao C, et al (2018) Core Structure and Electromagnetic Field Evaluation in WPT Systems for Charging Electric Vehicles. Energies 11:1-17.

https://doi.org/10.3390/en11071734 